\title{
Développement D'un Stock De Semences (Seedstocks) De L'algue Rouge Gelidium Corneum (Gelidiaceae, Rhodophyta)
}

\author{
Houda Chiheb \\ Groupe Algologie-Mycologie Appliquées, Laboratoire d'Ecologie, \\ Biodiversité et Environnement, Département de Biologie, Faculté des \\ Sciences de Tétouan, Université Abdelmalek Essaâdi, \\ M'hannech II, Tétouan, Maroc. \\ Département de Biologie, Faculté de sciences de la mer, Université de Las \\ Palmas de Gran Canaria, Juan de Quesada, Espagne

\section{Pilar García-Jiménez Rafael R. Robaina} \\ Département de Biologie, Faculté de sciences de la mer, Université de Las \\ Palmas de Gran Canaria, Juan de Quesada, Espagne

\section{Mustapha Hassoun Hassane Riadi} \\ Groupe Algologie-Mycologie Appliquées, Laboratoire d'Ecologie, \\ Biodiversité et Environnement, Département de Biologie, Faculté des \\ Sciences de Tétouan, Université Abdelmalek Essaâdi, \\ M'hannech II, Tétouan, Maroc.
}

Doi: 10.19044/esj.2018.v14n6p112 URL:http://dx.doi.org/10.19044/esj.2018.v14n6p112

\begin{abstract}
Gelidium corneum is a species of red algae notable for its commercial important as an agarophyte in Morocco. Several regions from the Moroccan Atlantic show that this alga is an endangered species due to the excessive tearing. Hence, the repopulation of these areas is necessary. The in vitro culture of the species was carried out in three media: enriched seawater medium (PES medium (Provasoli Enriched Seawater, Provasoli 1968)), medium with seawater (SW) and medium with artificial seawater, with the addition of polyamines (putrescine (put), spermidine (spd), and spermine (spr)) as a growth regulator in the three media. The results obtained are very significant, especially in PES medium with a growth rate of $95 \%$. Rhizoid formation and attachment of explants have been noted, especially in PES + Put medium.
\end{abstract}


Keywords: Agarophyte, Atlantic Coast, Gelidium corneum, in vitro culture, overexploitation

\section{Résumé}

La grande partie de la masse algale destinée à l'exportation est formée essentiellement par un mélange d'algues rouges de la famille des Gelidiaceae et dont la principale espèce reste l'agarophyte Gelidium corneum. L'exploitation du Gelidium corneum pour l'extraction de l'agar a considérablement augmenté dernièrement, ce qui a conduit à un épuisement remarquable des gisements de cette espèce. Entre 1999 et 2004 littoral marocain a perdu plus de $40 \%$ de ses réserves, dont la culture in vitro pourrait ouvrir de nouvelles possibilités pour repeupler les zones côtières endommagées. La culture in vitro de l'espèce a été effectuée dans trois milieux : milieu à base d'eau de mer enrichie (milieu PES (Provasoli Enriched Seawater, Provasoli 1968)), milieux avec l'eau de mer (SW) et milieu avec eau de mer artificielle, avec l'addition de polyamines (putrescine (put), spermidine (spd), et spermine (spr)) en tant que régulateur de croissance dans les trois milieux. Les résultats obtenus sont très significatifs surtout en milieu PES avec un taux de croissance de $95 \%$, de telle façon qu'on a remarqué la formation des rhizoïdes et la fixation des explants notamment en milieu PES + Put, alors que un taux de fixation de $80 \%$ a été obtenu dans le milieu SW contenant de la putrescine, et pour les boites de Pétri contenant de l'eau de mer artificielle aucune fixation $n^{6} a$ été signalé.

Mots clés: Gelidium corneum, agarophyte, surexploitation, côte atlantique, culture in vitro

\section{Introduction}

L'intérêt porté aux algues marines est en progression continue. elles sont connues depuis longtemps et constituent un important réservoir de molécules bioactives dont les domaines d'application sont nombreux. Ces algues étaient depuis longtemps utilisées en alimentation, en agro-alimentaire, cosmétique, pharmaceutique. Ces dernières années, elles font sujet de plusieurs recherches pharmacologiques et médicales (Chiheb et al. 2009; Bouhlal et al. 2010, 2011, 2013; De los Reyes et al. 2013; Zbakh et al. 2012, 2014; El Wahidi et al. 2015; Metidji et al. 2015; Boujaber et al. 2017).

Les algues constituent une part très importante de la biodiversité et une des base principale des réseaux trophiques des eaux douces, saumâtres et marines. Diverses espèces sont utilisées pour l'alimentation humaine, l'agriculture ou l'industrie (Bleakley et al. 2017). Elles contribuent ainsi au développement des activités socio-économiques mondiales. 
L'un des groupes les plus importants est celui des algues rouges dont on extrait du polygalactane dénommé agar, produit qui forme des gels utilisés dans plusieurs industries (Cole \& Sheath, 1990; Sebaaly et al. 2012; Balouiri, 2016; Hockett \& Baltrus, 2017).

L'exploitation des algues sur la côte atlantique marocaine a vu le jour durant la période de protectorat en 1949 à El Jadida, surtout la cueillette des algues rouges de la roche médiolittorale ou leur ramassage en épave (Riadi, 1998). L'intérêt devenait plus grand pour le Gelidium corneum (Hudson) J. V. Lamouroux dont les gisements sont importants entre El Jadida et Essaouira et son rendement en agar de $25 \%$ à $30 \%$ du poids sec (Sabour, 2012) est industriellement exploitable. L'agar est un polysaccharide pariétal des parois des algues rouges, un produit à forte valeur ajoutée, utilisé dans les domaines pharmaceutiques, cosmétiques, agro-alimentaires et pour divers usages, notamment dans des produits bio en vogue actuellement dans les pays développés (Villanueva et al. 2010).

L'exploitation du Gelidium corneum au Maroc a précédé toutes recherches scientifiques et malgré la réglementation, le volume de l'exploitation et d'exportation n'a cessé d'augmenter. Une des conséquences de l'arrachage manuelle accru est la régénération difficile de l'espèce, ce qui a entrainé une réduction des étendues initiales sur le médiolittoral et infralittoral supérieur. Son biotope est alors affecté d'où son confinement à des profondeurs plus grandes et par conséquent une nécessité de plonger pour l'atteindre (Riadi, 1998; Givernaud et al. 2005).

Malgré les intentions gouvernementales pour protéger l'espèce, les impacts causés représentent une réelle menace sur les richesses spécifiques et éco-systémiques. En effet, cette algues est la seule de la flore marine marocaine, que la loi essaye de protéger de l'intense exploitation destructive. Pour sa protection, deux arrêtés ont déjà été publiés (l'arrêté du 20 octobre 1950, BO N ${ }^{\circ} 1983$, et l'arrêté $n^{\circ} 1118-93$ publié le 1er décembre 1993, BO $\mathrm{N}^{\circ}$ 423) (ONEM, 2006).

Pour participer à la protection de ces algues, des études scientifiques de pointe doivent être entreprises notamment des études de culture in vitro et in vivo pour le repeuplement des zones atlantiques dans un contexte de développement durable.

La culture in vitro pourrait ouvrir de nouvelles possibilités pour repeupler les aires côtières endommagées. Ces techniques concernent les macroalgues marines dotées d'une valeur économique ou sociale et dont les stocks naturels sont insuffisants pour répondre à une demande croissante en produits de la mer notamment les phycocolloïdes.

Au Maroc, les études sur le Gelidium ont concerné les rendements en agar et la valorisation des résidus d'extraction (Ouhssine et al. 2006, 2007; Hanif et al. 2014). Vu la place économique et sociale de l'espèce, la présente 
recherche a été initiée dans le cadre du projet "Observatoire Marin Atlantique Iles Canaries-Maroc" (OMARAT).

\section{Matériel et Méthodes}

\section{Collecte de l'espèce et préparation des explants axéniques}

Le Gelidium corneum a été collectée sur la côte atlantique durant le mois de septembre 2011, à $15 \mathrm{~km}$ de la ville de Larache, sur la côte rocheuse de Lahyayda (35 ${ }^{\circ} 05^{\prime} 15.7$ "N, $\left.6^{\circ} 12^{\prime} 51.4^{\prime \prime} \mathrm{W}\right)$ (fig. 1). Les échantillons récoltés (fig. $2 \mathrm{~A}$ ) ont été nettoyés en premier à l'eau courante afin d'éliminer toutes les impuretés possibles (sels, sables et coquilles), puis on a procèdé à la suppression des épiphytes sous une loupe binoculaire à l'aide d'une brosse ou d'une spatule (Robaina et al. 1990a-b; García-Jiménez et al. 1999; Yong et al. 2011).

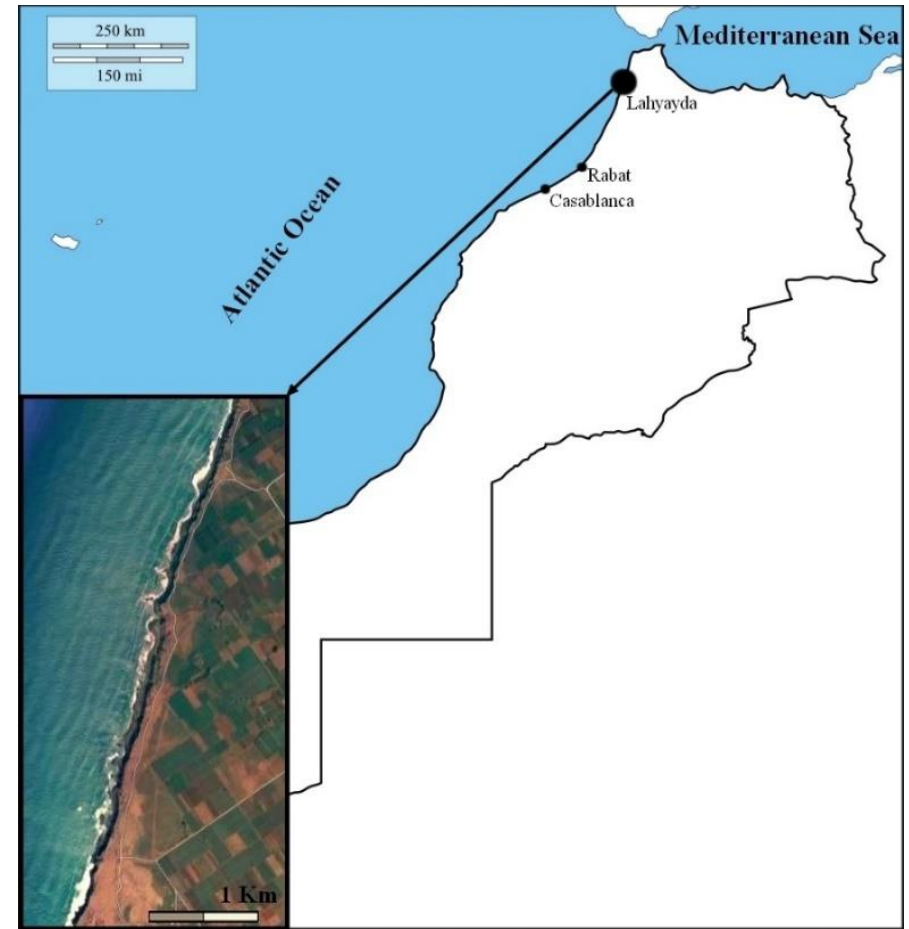

Figure 1: Carte géographique de la région du Lahyayda (Larache, Maroc)

Le matériel a été lavé par la suite, à l'eau de mer stérilisée jusqu'à l'obtention d'une matière propre. Les échantillons ont été mis dans des sacs en plastique et elles ont été ensuite transportées au département de biologie à l'université de Las Palmas des Iles Canaries à l'état frais dans un réfrigérateur.

Les 72 explants ont été prélevés, traités à l'eau de mer stérile, de la bétadine à $5 \%$ et une goutte de Tween 80 . Ils ont été par la suite mis dans un bain à ultrason durant 7 min puis lavés à l'eau de mer stérilisée afin d'éliminer 
toutes traces de la Bétadine. Après lavage, les explants ont été coupés, par une lame chirurgicale stérile, en segments de $1 \mathrm{~cm}$ de longueur (fig. $2 \mathrm{~B}$ ).

\section{Culture des explants}

Six explants axéniques (fig. 2 B) ont été déposés dans 4 boîtes de Pétri de $6 \mathrm{~cm}$ de diamètre contenant soit, le milieu PES (Provasoli et al. 1957), soit un milieu contenant de l'eau de mer artificielle, soit le milieu avec de l'eau de mer. Tous ces milieux sont additionnés de polyamines avec ou sans présence de mailles. L'expérience a été répétée 3 fois ( 24 x 3 fois $x$ traitement).

L'ensemble des boites est incubé dans une chambre de culture pendant une semaine à une température de $19 \pm 2{ }^{\circ} \mathrm{C}$. La photopériode est de 18 heures de lumière et 6 heures d'obscurité, l'intensité de la lumière blanche est de 30 $\mathrm{pE} \mathrm{m}^{-2} \mathrm{~S}^{-1}$ (Sylvania Grolux) (fig. 2C).

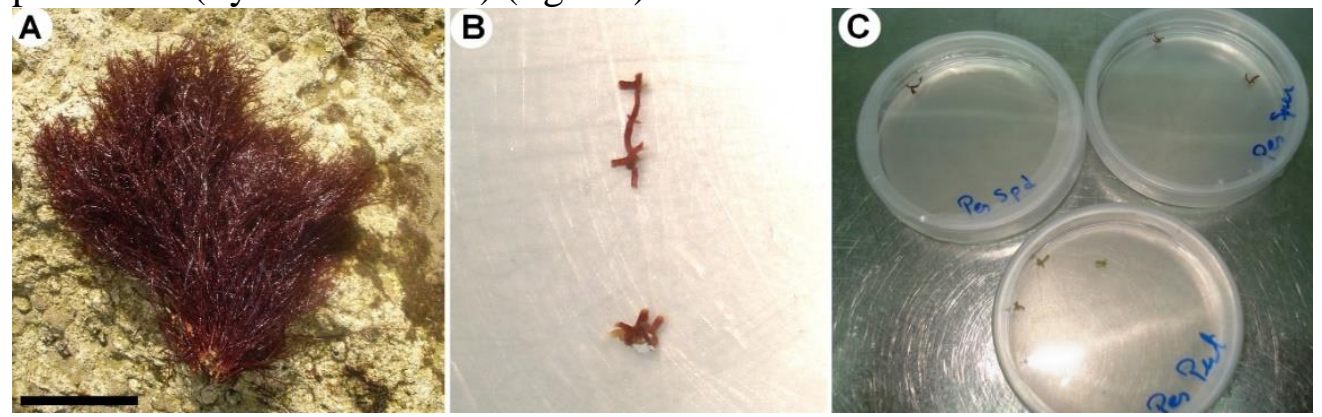

Figure 2 : A. Thalle de Gelidium corneum collecté sur la côte Atlantique marocaine, B. Les explants après la stérilisation, C. Les explants dans des boîtes de Pétri prêts à être cultivés.

Échelles : $\mathrm{A}=10 \mathrm{~cm}$

\section{Analyses statistiques des données}

Les données sur le taux de croissance des explants ont été testées pour la normalité et l'homogénéité de la variance en utilisant StatGraphics Centurion XVI.

Une analyse ANOVA a été réalisée pour déterminer le coefficient de corrélation $(\mathrm{P}<0,05)$ entre les milieux utilisés et les taux de croissance.

\section{Résultats}

Le taux de croissance spécifiques pour 24 explants répétés 3 fois avec ou sans présence de mailles à une température de $19 \pm 2{ }^{\circ} \mathrm{C}$ variés de $0 \%$ à $95 \%$. Une régénération des pousses a été observée à partir des extrémités des explants qui ont été cultivée dans les trois milieux : milieu à base d'eau de mer enrichie (milieu PES (Provasoli Enriched Seawater), milieu avec l'eau de mer (SW) et milieu avec eau de mer artificielle.

Les résultats obtenus sont résumés dans les tableaux 1, 2, 3. On remarque la formation des pousses et des pousses latérales (fig. 3 B) dans les différents milieux utilisés, notamment ceux qui contiennent les polyamines de 
types spermine et spermidine. Cependant, sur les milieux ne contenant que la putrescine, on observe la formation de rhizoïdes et la fixation des explants sur les mailles (fig. 3 A-C).

Des proliférations latérales ont été observées au niveau des extrémités des explants cultivés sur les milieux PES + Spd et PES + Spm durant les 7 jours suivant la culture et un pourcentage de $50 \%$ de régénération a été observé (fig. 3 B).
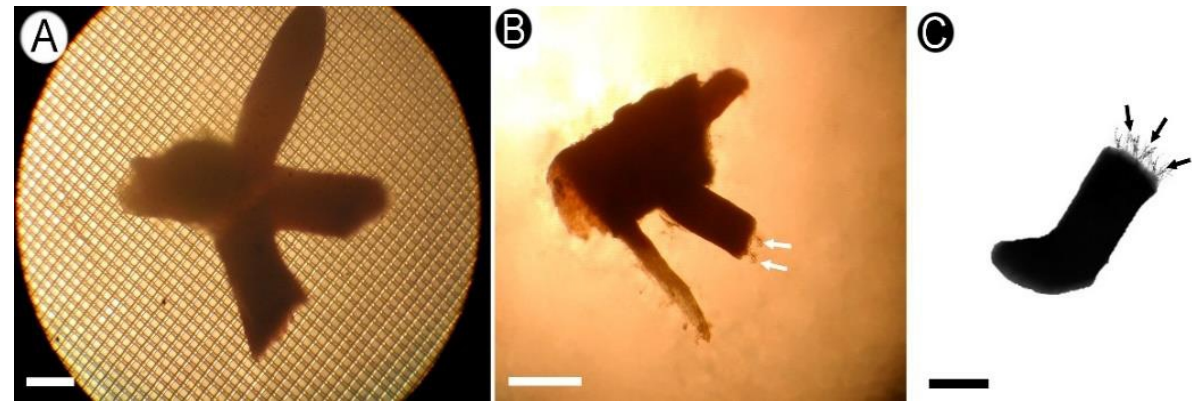

Figure 3 : A. Fixation de l'explant en présence de maille, B. Formation de pousses latérales (flèches), C. Formation des rhizoïdes (flèches). Échelles : A, B et C $=200 \mu \mathrm{m}$

Sur le milieu PES + Put, il est remarqué la formation des rhizoïdes et la fixation des explants sur les mailles (tab. 1), avec un pourcentage de régénération de $95 \%$.

La fixation des explants a été obtenue seulement dans les milieux (PES, SW) contenant la putrescine (tab. 1 et 2), alors qu'aucune fixation n'a été observée chez les explants cultivés sur les milieux ne contenant que de l'eau de mer artificielle (tab. 3).

Tableau 1: Résultats obtenus après l'utilisation de milieu PES additionné de polyamines (spermidine, putrescine, spermine) $(100 \%=72$ explants $)$

\begin{tabular}{|c|c|c|c|}
\hline Milieux & Mailles & Observations & $\begin{array}{c}\text { Croissance et fixation } \\
(\%)\end{array}$ \\
\hline PES+Spm $\left(10^{-3} \mathrm{M}\right)$ & + & Formation des pousses latérales & 50 \\
\hline PES+Put $\left(10^{-3} \mathrm{M}\right)$ & + & $\begin{array}{c}\text { Formation des rhizoïdes (Fixation } \\
\text { des explants })\end{array}$ & 95 \\
\hline PES+Spd $\left(10^{-3} \mathrm{M}\right)$ & + & $\begin{array}{c}\text { Formation des pousses + pousses } \\
\text { latérales }\end{array}$ & 50 \\
\hline
\end{tabular}

Mailles: Types de substrats de culture in vitro préalablement stérilisé, pour favoriser l'ancrage des rhizoïdes, + : Présence de mailles ; - : Absence de mailles

Tableau 2: Résultats obtenus après l'utilisation de milieux à base d'eau de mer additionnée de polyamines de type putrescine $(100 \%=72$ explants $)$

\begin{tabular}{|c|c|c|c|}
\hline Milieux & Mailles & Observations & $\begin{array}{c}\text { Croissance et fixation } \\
(\%)\end{array}$ \\
\hline $\mathrm{SW}+\mathrm{Spm}\left(10^{-3} \mathrm{M}\right)$ & + & Formation des pousses latérales & 50 \\
\hline $\mathrm{SW}+\mathrm{Spd}\left(10^{-3} \mathrm{M}\right)$ & + & Formation des pousses & 53 \\
\hline
\end{tabular}




\begin{tabular}{|c|c|c|c|}
\hline SW+Put $\left(10^{-3} \mathrm{M}\right)$ & + & $\begin{array}{c}\text { Formation des rhizoïdes (Fixation des } \\
\text { explants) }\end{array}$ & 80 \\
\hline $\mathrm{SW}+\mathrm{Spm}\left(10^{-3} \mathrm{M}\right)$ & - & Formation des pousses & 50 \\
\hline SW+Spd $\left(10^{-3} \mathrm{M}\right)$ & - & Formation des pousses latérales & 53 \\
\hline SW+Put $\left(10^{-3} \mathrm{M}\right)$ & - & Formation des rhizoïdes & 80 \\
\hline
\end{tabular}

Tableau 3: Résultats obtenus après l'utilisation de milieux contenant que de l'eau de mer artificielle additionnée de polyamines $(100 \%=72$ explants $)$

\begin{tabular}{|c|c|c|c|}
\hline Milieux & Mailles & Observations & $\begin{array}{c}\text { Croissance et } \\
\text { fixation }(\%)\end{array}$ \\
\hline $\begin{array}{c}\mathrm{H} 2 \mathrm{oD}+\mathrm{NaHCo} 3+\mathrm{CaCl} 2 \\
+\mathrm{Spd}\left(10^{-3} \mathrm{M}\right)\end{array}$ & + & Formation de pousses latérales & 1 \\
\hline $\begin{array}{c}\mathrm{H} 2 \mathrm{oD}+\mathrm{NaHCo}+\mathrm{CaCl} 2 \\
+\mathrm{Spm}\left(10^{-3} \mathrm{M}\right)\end{array}$ & + & Formation de pousses latérales & 1 \\
\hline $\begin{array}{c}\mathrm{H} 2 \mathrm{oD}+\mathrm{NaHCo}+\mathrm{CaCl} 2 \\
+\mathrm{Put}\left(10^{-3} \mathrm{M}\right)\end{array}$ & + & Formation de pousses latérales & 23 \\
\hline $\begin{array}{c}\mathrm{H} 2 \mathrm{oD}+\mathrm{NaHCo} 3+\mathrm{CaCl} 2 \\
+\mathrm{Spd}\left(10^{-3} \mathrm{M}\right)\end{array}$ & - & Formation de pousses latérales & 28 \\
\hline $\begin{array}{c}\mathrm{H} 2 \mathrm{oD}+\mathrm{NaHCo}+\mathrm{CaCl} 2 \\
+\mathrm{Spm}\left(10^{-3} \mathrm{M}\right)\end{array}$ & - & Formation de pousses latérales & 32 \\
\hline $\begin{array}{c}\mathrm{H} 2 \mathrm{oD}+\mathrm{NaHCo} 3+\mathrm{CaCl} 2 \\
+\mathrm{Put}\left(10^{-3} \mathrm{M}\right)\end{array}$ & - & Formation de pousses latérales & \\
\hline
\end{tabular}

\section{Discussion}

Nos essais de la culture in vitro du Gelidium corneum ont donné des résultats prometteurs. La composition chimique du milieu de culture et les régulateurs de croissance utilisés ont influencé la croissance des explants cultivés

\section{Composition chimique du milieu de culture}

Dans notre cas, le milieu de culture idéal pour la croissance et la régénération des explants de G. corneum est le PES avec lequel on a obtenu un taux de croissance de $95 \%$, en plus il a permis la formation des rhizoïdes et la fixation des explants notamment en milieu PES + Put. Ce résultat est similaire à ce qui a été obtenu par Yong et al. (2013) sur la propagation des Rhodophycées. Les mêmes résultats ont été signalés sur le même milieu par Jong et al. (2015) sur les explants de Gracilaria changii. Mais D'autres études se sont intéressées aux conditions optimales pour la croissance de l'espèce commerciale Kappaphycus alvarezii, algue rouge de l'ordre des Gigartinales, et ont montré que le milieu PES donne d'excellents résultats en le combinant avec différents paramètres (Baweja et al. 2009; Yong et al. 2011, 2013)

\section{Effet des régulateurs de croissance}

Les résultats que nous avons obtenus avec les régulateurs de croissance montrent que les polyamines de type spermine et spermidine favorisent la 
formation des pousses et pousses latérales alors que la putrescine favorise la rhizogenèse.

Les régulateurs de croissance, putresine, spermidine et la spermine, ont été testés sur la culture in vitro axénique de Grateloupia doryphora, spermine a induit la formation d'une masse désordonnée de cellules à partir du tissu organisé de carpospores, la putrescines et la spermidines ont transformé les carpospores en masse cellulaire qui a produit des pousses. La combinaison de la putrescine, spermidine et la spermine a conduit à une plus grande taille de la masse cellulaire qui a conduit à son tour à une plus grande quantité de pousses (García Jiménez et al. 1998; Marián et al. 2000; Sacramento et al. 2004). Le même résultat a été obtenu par Muñoz et al. (2006) sur la culture de Kappaphycus alvarezii.

Le rôle des polyamines dans ce travail était de contribuer à l'adhésion des algues au substrat, étant donné de la nature polycationique de ces molécules qui favorisent l'interaction des groupes sulfatés et neutres des polysaccharides constitutifs du mucilage et les parois cellulaires des algues (García Jiménez et al. 1998). Les résultats de ce travail nous ont permis de discerner les traitements qui favorisent la rhizogenèse, et le développement des pousses latéraux en une période très courtes.

\section{Conclusion}

Les expériences ménées dans cette étude, ont permis d'avoir une certitude sur les traitements qui favorisent la rhizogenèse, le développement de pousses et de pousses latérales chez le Gelidium corneum. Dans la présente étude, la plus forte prolifération a été observée dans les milieux qui contient du putrescine dans la semaine suivant la culture et s'est avérée meilleure comparativement aux autres milieux où on a remarqué la prolifération des pousses.

Cette étude a permis de conclure que la composition chimique de milieu additionné de régulateurs de croissance de types putrescine, spermidine et spermine, et l'utilisation d'explants axéniques viables permettent l'obtention de plantules avec des rhizoïdes, des pousses et des pousses latérales.

\section{References:}

1. CHIHEB, I., H. RIADI, J. MARTINEZ-LOPEZ, J.F. DOMINGUEZ SEGLAR, J.A. GOMEZ VIDAL, H. BOUZIANE \& M. KADIRI 2009- Screening of antibacterial activity in marine green and brown macroalgae from the coast of Morocco. Afr. J. Biotechnol. 8(7): 12581262. 
2. BOUHLAL, R., H RIADI \& N. BOURGOUGNON -2010- Antiviral activities of Morocco seaweeds extracts. Afr. J. Biotechnol. 9: 79687975.

3. BOUHLAL, R., C. HASLIN, J.C. CHERMANN, S. COLLIECJOUAULT, C. SINQUIN, G. SIMON, S. CERANTOLA, H. RIADI \& N. BOURGOUGNON -2011- Antiviral activities of sulfated polysaccharides isolated from Sphaerococcus coronopifolius (Rhodophytha, Gigartinales) and Boergeseniella thuyoides (Rhodophyta, Ceramiales). Mar. Drug. 9(7): 1187-1209.

4. BOUHLAL, R., H. RIADI \& N. BOURGOUGNON -2013Antibacteriala of the extracts of Rhodophyceae from the Atlantic and the Mediterranean coasts of Morocco. J. Microbiol Biotechnol. Food Sci. 2(6): 2431-2439.

5. DE LOS REYES, C., ZBAKH, H., V. MOTILVA \& E. ZUBÍA -2013Antioxidant and anti-inflammatory meroterpenes from the brown alga Cystoseira usneoides. J. Nat. Product. 76(4): 621-629.

6. ZBAKH, H., CHIHEB, H., BOUZIANE, H., V. MOTILVA \& H. RIADI -2012- Antibacterial Activity of Benthic Marine Algae Extracts from the Mediterranean Coast of Morocco. J. Microbiol., Biotechnol. Food Sci. 2(1): 219-228.

7. ZBAKH, H., CHIHEB, I., V. MOTILVA \& H. RIADI -2014Antibacterial, Cytotoxic and Antioxidant Potentials of Cladophora prolifera (Roth) Kutzing extract from the Mediterranean coast of Morocco. Am. J. Phytomed. Clin. Ther. 2 (10): 1187-1199.

8. EL WAHIDI, M., EL AMRAOUI, B., M. EL AMRAOUI \& T. BAMHAOUD -2015- Screening of antimicrobial activity of macroalgae extracts from the Moroccan Atlantic coast. Ann Pharm, 73(3):190-196. doi: 10.1016/j.

9. METIDJI H., DOB T., TOUMI M., KRIMAT S., A. KSOURI \& A. NOUASRI -2015- In vitro screening of secondary metabolites and evaluationof antioxidant, antimicrobial and cytotoxic properties of Gelidium sesquipedale Thuret et Bornet red seaweed from Algeria. J. Mater. Environ. Sci. 6(11): 3184-3196.

10. BOUJABER, N, OUMASKOUR, K, O. ASSOBHEI \& S. ETAHIRI 2017-Antimicrobial activity of different fractions obtained from Gelidium sesquipedale and Laminaria ochroleuca. J. Bio. Innov. 6(3): 306-312.

11. BLEAKLEY, S. \& M. HAYES -2017-. Algal Proteins: Extraction, Application, and Challenges Concerning Production. Foods, 6(5), 33.

12. COLE, K.M. \& R.G. SHEATH -1990- Biology of the red algae. Cambridge University Press, Cambridge, 517 pp. 
13. SEBAALY, C., KARAKI, N., CHAHINE, N., EVIDENTE, A., YASSINE A., J. HABIB \& H. KANAAN -2012- Polysaccharides of the red algae "Pterocladia" growing on the Lebanese coast: Isolation, structural features with antioxidant and anticoagulant activities. J. App. Pharm. Sci. 2(10): 1-10.

14. BALOUIRI, M., M. SADIKI \& S. K. IBNSOUDA -2016- Methods for in vitro evaluating antimicrobial activity: A review. J. Pharmac. Anal. 6(2): 71-79.

15. HOCKETT, K. L. \& D. A. BALTRUS -2017- Use of the Soft-agar Overlay Technique to Screen for Bacterially Produced Inhibitory Compounds. J. Visual. Exper: JoVE, (119). doi:10.3791/55064.

16. RIADI, H. -1998- Biodiversité des algues et phytoplancton marin du Maroc. Rapport biodiversité au Maroc. Programme des nations unies pour l'environnement (PNUE). Ministère de l'environnement du Maroc.

17. SABOUR, B. -2012- L'algue rouge agarophyte Gelidium sesquipedale ou 'rbiâa' de la côte des Doukkala. Ed. Livre des Prestige de la ville d'El Jadida. 38 p.

18. VILLANUEVA, R.D., SOUSA, A.M.M., GONÇALVES, M.P., M. NILSSON \& L. HILLIOU -2010- Production and properties of agar from the invasive marine alga, Gracilaria vermiculophylla (Gracilariales, Rhodophyta). J. Appl. Phycol. 22: 211-220.

19. GIVERNAUD, TH., SQALI, N., BARBAROUX, O., ORBI, A., SEMMAOUI, Y., REZZOUM, N., A. MOURADI \& R. KAAS -2005Mapping and biomass estimation for a harvested population of Gelidium sesquipedale (Turn.) Thuret (Rhodophyta, Gelidiales) along the Atlantic coast of Morocco. Phycologia 44(1): 66-71.

20. ONEM. -2006 - Étude Nationale sur la Biodiversité. Algues Marines. Observatoire National de l'Environnement du Maroc, 95.

21. OUHSSINE. K., M. OUHSSINE \& M. EL YACHIOUI -2006Caractérisation chimique et microbiologique des déchets de Gelidium sesquipedale avant et après fermentation. Bull. Soc. Pharm. Bordeaux, 145: 31-40.

22. OUHSSINE. K., M. OUHSSINE \& M. EL YACHIOUI -2006L'application des déchets traités de l'algue Gelidium sesquipédale dans la culture du Maïs. Afr. Sci. 03(2): $259-270$.

23. HANIF N., CHAIR M., M.C. IDRISSI \& T. NAOKI -2014L'exploitation des algues rouges Gelidium dans la région d'El-Jadida: Aspects socio-économiques et perspectives. Afr. Sci. 10(1): 103-126.

24. ROBAINA, R.R., P. GARCÍA-JIMÉNEZ \& A. LUQUE -1990aMorphogenetic effect of glycerol on tissue cultures of the red seaweed Grateloupia doryphora. J. Appl. Phycol. 2: 137-143. 
25. ROBAINA, R.R., G. REINA \& A. LUQUE -1990b- The effects of the physical characteristics of the culture medium on the development of red seaweeds in tissue culture. Hydrobiologia 204/205: 137 - 142.

26. GARCÍA-JIMÉNEZ, P., MARIÁN, F.D., M. RODRIGO \& R.R. ROBAINA -1999- Sporulation and sterilization method for axenic culture of Gelidium canariensis. J. Biotech. 70: 227-229.

27. YONG, W.T.L., TING, S.H., CHIN, W.L., K.F. RODRIGUES \& A. ANTON -2011- In vitro Micropropagation of Eucheuma Seaweeds. In: International Conference on Biotechnology and Food Science (ICBFS), 01-03 April 2011, Bali Island, Indonesia.

28. PROVASOLI, L., J.A. MCLAUGHLIN \& M.R. DROOP -1957- The development of artificial media for marine algae. Arch. Microbiol. 25(4): 392-428.

29. YONG, Y.C. \& J.J. ZHONG -2013- Regulation of aromatics biodegradation by rhl quorum sensing system through induction of catechol meta-cleavage pathway. Bio. Tech. 136: 761-765.

30. JONG, L.W., V.Y. THIEN, Y.S. YONG, K.F. RODRIGUES \& W.T.L. YONG -2015- Micropropagation and protein profile analysis by SDS-PAGE of Gracilaria changii (Rhodophyta, Solieriaceae). Aqu. Rep. 1: 10-14.

31. BAWEJA, P. \& D. SAHOO -2009- Regeneration Studies in Grateloupia filicina (J.V. Lamouroux) C. Agardh - An Important Carrageenophyte and Edible Seaweed. Algae 24(3): 163-168.

32. GARCIA JIMENEZ, P., M. RODRIGO \& R. ROBAINA -1998Influence of plant growth regulators, polyamines and glycerol interaction on growth and morphogenesis of carposporelings of Grateloupia cultured in vitro. J. App. Phyc. 10: 95-100.

33. MARIÁN, F.D., P. GARCÍA-JIMÉNEZM \& R.R. ROBAINA -2000Polyamines in marine macroalgae, levels of putrescine, Espermidine and spermine in thalli and changes in their concentration during glycerol-induced cell growth in vitro. Physiol. Plant. 110: 530 -534.

34. SACRAMENTO, A.T., P. GARCÍA-JIMÉNEZ \& R. ROBAINA 2004- Influence of polyamines on the sporulation of Grateloupia (Halymeniaceae, Rhodophyta). J. Phycol. 40: 887-894.

35. MUÑOZ, J., A.C. CAHUE-LÓPEZ, R. PATIÑO \& D. ROBLEDO 2006- Use of plant growth regulators in micropropagation of Kappaphycus alvarezii (Doty) in airlift bioreactors. J. App. Phycol. 18(2): 209-218.

36. PREECE, J.E. -1995- Can nutrient salts partially substitute for plant growth regulators. Pl. Tissue Cult. Biotech. 1: 26-37.

37. CARL, M.R. \& R.W. RICHARD -2002- Mineral nutrition and plant morphogenesis. In Vitr. Cell. Dev. Bio - Plant 38: 116-124. 
38. MAALEJ, M., A. R. CHAARI \& N. DRIRA -2006- Contribution to the improvement of olive tree somatic embryogenesis by mineral and organic analysis of zygotic embryos. Euphytica 151: 31-37.

39. KARIMI, G., M. GHORBANLI, H. HEIDARI, R.A. KHAVARINEJAD \& M.H. ASSAREH -2005- The effects of $\mathrm{NaCl}$ on growth, water relations, osmolytes and ion content in Kochia prostrata. Biol. Plant., 49(2): 301-304.

40. GOLDSTEIN, M.E. -1973- Regeneration and vegetative propagation of the agarophyte Gracilaria deblis (Forsskal) Borgesen (Rhodophyceae). Bot. Mar. 16: 226-228.

41. BULA-MEYER, G. -1989 - Experimental culture in the sea of the red macroalgae Grateloupia filicina. In: de Oliveira E. C. \& N. Kautsky (Eds.), pp. 101-104. Cultivation of Seaweeds in Latin American. Sau Sebastiao, Brazil.

42. HURTADO-PONCE, A.Q. -1990- Vertical rope cultivation of Gracilaria (Rhodophyta) using vegetative fragments. Bot. Mar. 133: 477-481.

43. GARCÍA-JIMÉNEZ, P., M. RODRIGO \& R. ROBAINA -1998Influence of plant growth regulators, polyamines and glycerol interaction on growth and morphogenesis of carposporelings of Grateloupia cultured in vitro. J. Appl. Phycol. 10:95-100.

44. BAWEJA, P. \& D. SAHOO -2009- Regeneration Studies in Grateloupia filicina (J.V. Lamouroux) C. Agardh - An Important Carrageenophyte and Edible Seaweed. Algae 24(3): 163-168. 